\title{
Umwelt - Allergie - Asthma - ein Überblick
}

\author{
ERIKA VON MUTIUS
}

Es gibt zahlreiche Hinweise dafür, dass Umweltfaktoren im weitesten Sinn eine entscheidende Rolle bei der Entstehung von Asthma bronchiale und Allergien spielen. Allein schon die große Variabilität unter genetisch eng verwandten Populationen deutet auf die überragende Bedeutung von Umweltfaktoren hin.

Für die Entwicklung asthmatischer und allergischer Erkrankungen werden Risikofaktoren und protektive Faktoren diskutiert: An Risikofaktoren sind genetische Faktoren bedeutsam (positive Familienanamnese, Kopplungsstudien), das Geschlecht spielt eine Rolle (während der Kindheit ist das Asthma bei Buben häufiger, im Erwachsenenalter ist die Relation umgekehrt). Stillen senkt nur in den ersten drei Lebensjahren die Inzidenz atopischer Sensibilisierungen und obstruktiver Bronchitiden. In zahlreichen Studien ist ein inverser $\mathrm{Zu}$ sammenhang zwischen der Inzidenz von Atemwegsinfektionen und dem Vorkommen von Asthma beschrieben worden. Passivrauchexposition geht mit erhöhten Asthmahäufigkeiten einher.
Trotz positiver klinisch-experimenteller Daten gibt es derzeit nur wenig Anhaltspunkte dafür, daß eine erhöhte Ozonbelastung für die Entwicklung von Asthma oder Atopie verantwortlich ist. In der letzten Zeit rücken vielfach protektive Faktoren in den Vordergrund des wissenschaftlichen Interesses: Aufwachsen in großen Familien, frühkindlicher Kinderkrippen-Besuch, Vorhandensein älterer Geschwister, niedriger elterlicher Sozialstatus, frühkindliche Exposition gegenüber Haustieren, anthroposophischer Lebensstil, Aufwachsen auf einem landwirtschaftlichen Betrieb mit Tierhaltung. Insbesondere zu letzterem Thema liegt eine Fülle epidemiologischer und tierexperimenteller Daten vor, deren Synopsis faszinierend ist.

\section{Priv.-Doz. Dr. Erika von Mutius \\ Dr. von Haunersche Kinderklinik der \\ Universität München \\ Lindwurmstraße 4 \\ 80337 München}

\section{Bedeutung der Endotoxine für Allergien und Atemwegserkrankungen}

\author{
DENNIS NOWAK
}

Endotoxine sind Bestandteile der Zellwand gramnegativer Bakterien. Endotoxine lösen eine neutrophile Atemwegsentzündung aus und spielen eine Schlüsselrolle bei der Bronchitis nach Exposition gegenüber organischen Stäuben wie auch beim Organic Dust Toxic Syndrome - einem fieberhaften grippeähnlichen Krankheitsbild, welches ebenfalls nach Exposition gegenüber organischen Stäuben auftritt. Endotoxine haben möglicherweise auch eine pathophysiologische Bedeutung für die Entstehung der chronischen obstruktiven Bronchitis. Der Ausprägungsgrad allergischer asthmatischer Erkrankungen kann durch hohe Endotoxinexpositionen ungünstig beeinflusst werden.

Auf der anderen Seite stehen Beobachtungen, dass Kinder, die auf einem Bauernhof aufwachsen, also in einem Milieu mit nachgewiesener hoher Endotoxinbelastung, ein wesentlich geringeres $\mathrm{Ri}$ siko haben, eine Allergie, einen Heuschnupfen oder ein Asthma bronchiale zu entwickeln. Die Reduzierung des Risikos ist besonders ausgeprägt, wenn sich die Kinder in ihrem ersten Lebensjahr bereits regelmäßig im Stall aufhalten. Die wahrscheinlichste Erklärung dafür ist, dass ein hoher Gehalt an mikrobiellen Substanzen wie den Endo- 